\title{
Médiévales
}

Langues, Textes, Histoire

71 | automne 2016

Conflits et concurrence de normes

\section{Alcuin, Charlemagne et le droit d'asile}

Alcuin, Charlemagne and Sanctuary

\section{Maxence Bidu}

\section{(2) OpenEdition}

Journals

Édition électronique

URL : https://journals.openedition.org/medievales/7969

DOI : 10.4000/medievales.7969

ISSN : $1777-5892$

\section{Éditeur}

Presses universitaires de Vincennes

\section{Édition imprimée}

Date de publication : 20 novembre 2016

Pagination : $109-136$

ISBN : 978-2-84292-565-9

ISSN : 0751-2708

\section{Référence électronique}

Maxence Bidu, «Alcuin, Charlemagne et le droit d'asile », Médiévales [En ligne], 71 | automne 2016, mis en ligne le 20 novembre 2018, consulté le 24 avril 2022. URL : http://journals.openedition.org/ medievales/7969; DOI : https://doi.org/10.4000/medievales.7969 


\section{Maxence Bidu}

\section{Alcuin, Charlemagne et le droit d'asile}

Au tournant des années 801 et 802 , un clerc se présenta aux portes de l'abbaye Saint-Martin de Tours, sollicitant hébergement et protection auprès de l'abbé Alcuin. Il était originaire d'Orléans où, sur décision de l'évêque Théodulfe, on le retenait prisonnier pour ses fautes, jusqu'à ce qu'il s'évadât à la faveur de circonstances inconnues. De manière inattendue, son recours au droit d'asile constitua le point d'origine d'une querelle retentissante entre l'évêque d'Orléans et l'abbé de Saint-Martin de Tours. La qualité des deux prélats leur permit de requérir l'arbitrage direct de Charlemagne, l'un comme l'autre plaidant auprès de l'empereur pour obtenir son soutien.

L'intense débat qui fit rage par voie épistolaire fut l'occasion pour les deux camps d'exposer leurs arguments ; il révéla également l'ampleur de leur désaccord.

Cette correspondance revêt une importance capitale pour l'histoire du droit d'asile à l'époque carolingienne, car elle livre un témoignage unique de la réception des anciennes lois sur l'asile par les cadres de l'Empire et met en lumière l'emploi de ces lois dans la résolution d'un cas pratique.

Ce corpus documentaire a déjà fait l'objet de plusieurs études ${ }^{1}$. Luitpold Wallach, notamment, s'est intéressé à cet échange pour examiner la diffusion du droit romain à l'époque carolingienne. Plus récemment, Rob Meens a voulu inscrire son analyse de l'affaire dans l'historiographie du règlement des conflits au haut Moyen Âge, en cherchant à mettre en avant le rôle de la pénitence, via le recours à l'asile, dans le rétablissement de la paix.

1. P. Timbal, Le Droit d'asile, Paris, 1939, p. 181-182 ; L. Wallach, Alcuin and Charlemagne, Studies in Carolingian History and Literature, Ithaca (N.Y.), 1959, p. 99$140 ;$ H. Noizet, « Alcuin contre Théodulphe : un conflit producteur de normes », Annales de Bretagne et des pays de l'Ouest, 111/3 (2004), p. 113-129 ; R. MeEns, « Sanctuary, Penance, and Dispute Settlement under Charlemagne : The Conflict between Alcuin and Theodulf of Orléans over a Sinful Cleric », Speculum, 82/2 (2007), p. 277-300. 
En 2004, Hélène Noizet a proposé de s'appuyer sur cette correspondance pour illustrer le processus d'élaboration du droit au moment de l'accession de Charlemagne à la dignité impériale. Postulant l'influence de la pratique sur la production des normes, elle a considéré l'affaire de Tours comme un exemple de cas concret déclenchant une réaction législative de l'empereur, sous la forme d'une redéfinition du droit d'asile. Il ne s'agit pas, dans les lignes qui suivent, de contester l'idée selon laquelle la pratique et le contexte de production des lois influent sur leur orientation ${ }^{2}$. Il nous est cependant apparu que l'affaire de 801-802 n'est peut-être pas le meilleur exemple pour rendre compte de ce mécanisme. Il n'est pas certain en effet que le droit d'asile ait subi une transformation radicale à cette occasion.

En reprenant l'analyse parallèle des lettres et de la législation, il s'agit de questionner à nouveau le rapport des protagonistes au contenu même des lois, en identifiant quels textes sont invoqués et de quelle manière, en soulignant les dispositions qui sont retenues, rejetées ou ignorées, en évaluant, enfin, la part réelle des altérations, des modifications ou des innovations. Cela doit nous permettre de mieux cerner ce que cette affaire révèle de la perception de l'institution de l'asile sous le règne de Charlemagne et de réévaluer l'impact d'un tel conflit sur la législation ultérieure.

\section{Les sources de la querelle}

Le récit de l'affaire est longuement détaillé dans les travaux de L. Wallach et R. Meens. La querelle est connue par cinq lettres ${ }^{3}$, qui permettent de supposer la rédaction de sept autres documents connexes, malheureusement disparus ${ }^{4}$. Elles livrent le point de vue de deux des protagonistes : celui d'Alcuin, auteur de quatre missives sur les cinq, et celui de Charlemagne, dont la connaissance du cas se fonde sur les rapports qu'il reçut de l'abbé de Saint-Martin et de l'évêque d'Orléans.

Charlemagne fut rapidement informé de l'évasion du clerc par Théodulfe et autorisa la saisie du fugitif ${ }^{5}$. Alcuin assure que, dans un premier temps, l'individu avait bien été remis aux hommes de Théodulfe, pour qu'ils le conduisent auprès de l'empereur, comme le réfugié en avait fait la demande ${ }^{6}$. L'abbé affirme cependant que les membres de cette escorte décidèrent alors, d'eux-mêmes, de remettre le transfert à plus

2. Considérer les conventions juridiques comme le produit « d'une renégociation permanente entre les acteurs en fonction de leurs préoccupations et de leur vie concrète » nous paraît tout à fait pertinent : H. NOIZET, «Alcuin contre Théodulphe... », p. 127.

3. Alcuin, Ep. 245-249 (éd. E. Duemmler, MGH, Epistolae, IV, Berlin, 1895, p. 393-404). Désignées ci-après comme les lettres 245 à 249 .

4. L. Wallach, Alcuin and Charlemagne..., p. 99-101.

5. Alcuin, Lettre 247, p. 400.

6. Alcuin, Lettre 245, p. 393, et Lettre 246, p. 398. 
tard, invoquant leur crainte d'une embuscade tendue sur le chemin du retour. Le dimanche suivant, ils se présentèrent de nouveau aux portes du sanctuaire, plus nombreux et accompagnés de l'évêque de Tours, Joseph. C'est alors que la situation dégénéra : huit hommes de l'escorte pénétrèrent de force dans l'église et cherchèrent à arracher le réfugié de l'autel. Alertés par la rumeur d'un assaut mené sur leur sanctuaire, des mendiants (mendici) se ruèrent selon Alcuin sur les hommes de Théodulfe. Les moines de SaintMartin accoururent alors du réfectoire, s'employant autant qu'ils le purent à calmer la rixe.

Théodulfe et Alcuin écrivirent chacun à Charlemagne pour lui rapporter leur version des faits. Les deux lettres sont perdues, mais le contenu de celle d'Alcuin nous est connu car l'abbé envoya des rapports similaires à certains de ses proches : le premier à ses disciples Fridugise et $\mathrm{Wizo}^{7}$, qui résidaient à la cour et devaient y défendre sa position, le second à un évêque inconnu, peut-être Hildebold de Cologne ou Arn de Salzbourg ${ }^{8}$. Alcuin y relate les faits survenus, mais cherche surtout à justifier la résistance des moines à la saisie du réfugié en la fondant sur un ensemble de références bibliques et juridiques, scrupuleusement recensées pour l'occasion. Une telle compilation fait tout l'intérêt de ce rapport, car elle donne à voir l'état de la législation de l'asile telle que peut la connaître un grand abbé du début du IX $\mathrm{X}^{\mathrm{e}}$ siècle.

La réponse de Charlemagne à Alcuin est formulée en des termes froids et durs ${ }^{9}$. Faisant fi du plaidoyer de son abbé, l'empereur l'informe de l'envoi prochain d'un missus spécial, un certain Téotbert, chargé de mener son enquête et de tenir un plaid au nom de l'empereur ${ }^{10}$.

L'issue de cette enquête est connue par une ultime missive d'Alcuin à Charlemagne ${ }^{11}$. Après dix-neuf jours d'investigations, des moines de SaintMartin furent effectivement convaincus d'incitation à la révolte et reçurent des peines de flagellation et de mise aux fers ${ }^{12}$. L'issue défavorable de l'enquête amène l'abbé à reconnaître à mots couverts la possible implication

7. Désignés sous les noms de Nathanaël et Candide : Alcuin, Lettre 245, p. 393.

8. Alcuin, Lettre 246, p. 398.

9. Alcuin, Lettre 247, p. 399.

10. Alcuin, Lettre 249, p. 402.

11. Ibid., p. 401.

12. Dans cette même lettre, l'abbé tourangeau se plaint des méthodes employées par le missus. Oublieux des réformes apportées par Charlemagne aux procédures judiciaires, Alcuin dénonce l'impossibilité qui lui fut faite de présenter ses témoins et sa version des faits au procès. C'est que, conformément à une procédure d'enquête naissante, Téotbert sélectionna lui-même, parmi les boni homines, les individus qui durent témoigner et forgea son verdict en fonction de ces dépositions, selon des modalités bien différentes de la procédure accusatoire qu'Alcuin semblait attendre. F.-L. Ganshof ajoute qu'au plaid général d'octobre 802 les ecclésiastiques réclamèrent le droit de recourir à la procédure d'enquête, jusque-là essentiellement employée par le palais et les missi : F.-L. GANSHOF, « Charlemagne et l'administration de la justice dans la monarchie franque », dans H. Beumann éd., Karl der Grosse. Lebenswerk und Nachleben, vol. 1, Düsseldorf, 1965, p. 394-419 (p. 414-415). 
de certains moines dans le déclenchement de la rixe, mais c'est pour mieux insister sur le fait que cette dernière n'est que la conséquence de fautes antérieures dont la responsabilité est tout entière rejetée sur Théodulfe et ses hommes ${ }^{13}$. Pour se dédouaner, Alcuin use de procédés rhétoriques d'autant plus faciles à détecter qu'ils sont, comme l'a montré Luitpold Wallach, répertoriés dans son propre traité, le De rhetorica, inspiré du De inventione de Cicéron ${ }^{14}$. Contre les conclusions du procès, Alcuin réitère donc ses accusations vis-à-vis de l'évêque d'Orléans, quitte à aggraver toujours plus l'état de ses relations avec l'empereur. L'issue de l'affaire ne nous est cependant pas connue.

\section{La défense du droit d'asile par Alcuin}

La lettre envoyée par Alcuin à ses proches demeurant à la cour impériale présente un intérêt particulier car l'abbé y fournit un argumentaire étoffé qui doit leur servir à plaider pour le soutenir dans sa défense du droit d'asile ${ }^{15}$. La quasi-totalité des sources invoquées par cette lettre ont été identifiées par Ernst Dümmler pour les Monumenta Germaniae Historica ${ }^{16}$. L'attribution par Alcuin de deux canons à un prétendu concile d'Agde a été rectifiée dès l'ouvrage de Pierre Timbal ${ }^{17}$, qui a montré qu'il s'agissait en réalité de textes apocryphes extraits par l'abbé de la Collectio Hibernensis ${ }^{18}$. Alcuin puise ainsi ses références conciliaires dans les multiples collections canoniques qui circulent au haut Moyen $\hat{A} g \mathrm{e}^{19}$. Il s'appuie aussi largement

13. Dans sa thèse soutenue en 2013, Claire Tignolet indique que les relations entre Alcuin et Théodulfe étaient empreintes de rivalité, voire d'animosité (C. TIGNOLET, Exsul et exsul erat. Théodulfe (vers 760-820/821) : parcours biographique, thèse de doctorat en histoire, sous la direction de Régine LE JAN, Université Paris 1 - Panthéon Sorbonne, 2013, p. 370-377). L'abbé de Tours n'aurait ainsi pas jugé nécessaire de conserver les lettres produites à l'occasion de cette querelle, par lui et ses correspondants, en raison de son issue défavorable (p. 164).

14. Rejeter la faute sur autrui ou souligner une faute antérieure causée par la partie adverse (AlcuIn, Disputatio de rhetorica et de virtutibus, cap. XIV, éd. W. S. Howell, The Rhetoric of Alcuin and Charlemagne, Princeton, 1941, p. 84) ; nier l'intention du crime, celui-ci étant décrit comme le fruit de l'ignorance, du hasard ou de la nécessité (cap. XV, p. 88) ; prouver qu'on mène une vie honorable et qu'on ne peut donc être coupable des faits reprochés, ou que ceux-ci furent produits par l'ignorance, par accident ou à l'instigation d'autrui (cap. XXVI, p. 108-110).

15. Alcuin, Lettre 245, p. 393.

16. Ibid., p. 393-398.

17. P. Timbal, Le Droit d'asile, p. 180-181.

18. Compilée en Irlande dans la première moitié du VIII ${ }^{\mathrm{e}}$ siècle : L. KéRY, Canonical Collections of the Early Middle Ages (400-1140), Washington (D.C.), 1999, p. 73. Voir aussi H. Wasserschleben, Die Irische Kanonensammlung, Leipzig, 1885.

19. Sur ces collections, consulter : F. MAASSEN, Geschichte der Quellen und der Literatur des Canonischen Rechts im Abendlande, Graz, 1870, p. 204-209 ; Les Canons des conciles mérovingiens, $v I^{e}$-VII ${ }^{e}$ siècles, éd. J. Gaudemet et B. BASDEvant, Paris, t. I, 1989, p. 14-16 ; L. KéRY, Canonical Collections..., p. 43-50. À partir du VII siècle, une partie des canons de ces conciles se transmet plus largement via la collection dite Hispana, dont la première version 
sur le droit romain et cite plusieurs interpretationes issues du Bréviaire d'Alaric ${ }^{20}$. L'abbé présente ses sources par ordre d'autorité, en plaçant la Bible avant les canons conciliaires et le droit romain. Son argumentaire vise à démontrer que la justice des hommes doit être rendue avec miséricorde et que l'institution de l'asile doit favoriser l'expression de cette clémence en contenant la violence par un ensemble de procédures, lesquelles n'auraient pas été respectées dans le cas du fugitif d'Orléans.

\section{Nécessité de la miséricorde et du pardon dans la mise en œuvre de la justice}

La supériorité de la loi divine sur toute loi humaine étant admise, Alcuin entreprend dans un premier temps de fonder la défense du clerc réfugié sur un ensemble de passages de la $\mathrm{Bible}^{21}$, selon une logique typique de la renaissance carolingienne, qui cherche à fonder le salut du peuple chrétien sur une bonne connaissance de l'Écriture et sur l'adéquation de la justice terrestre avec les préceptes divins ${ }^{22}$.

À l'issue de son exposé, l'idée de miséricorde apparaît comme la première justification du droit des pécheurs à se réfugier dans les églises pour y être protégés. Puisque tout homme connaît le péchée ${ }^{23}$, chacun doit pouvoir, à tout moment, pénétrer dans le temple de Dieu pour s'y confesser, se repentir et y recevoir miséricorde et pardon ${ }^{24}$. En tout état de cause, la miséricorde doit prévaloir sur le jugement ${ }^{25}$. Il ne s'agit donc pas pour Alcuin de trouver dans les Écritures une légitimation explicite du droit d'asile, mais de démontrer que le principe sur lequel celui-ci repose y est présenté comme un devoir fondamental des chrétiens.

Bien qu'il ne se réfère à aucun auteur particulier, nous pouvons remarquer avec intérêt que l'abbé de Saint-Martin adopte une logique analogue à celle des plaidoyers en faveur du droit d'asile élaborés au tournant des $I V^{e}$ et $v^{e}$ siècle par Jean Chrysostome et Augustin. Le premier, dans une

voit le jour en Espagne vers 633-636. Elle est progressivement complétée et est diffusée sous diverses formes au sein de la Gaule franque, jusqu'au cœur du IX ${ }^{\mathrm{e}}$ siècle puisqu'elle est employée par les auteurs des recueils pseudo-isidoriens. G. Martínez Dízz, La Colección canónica Hispana, Madrid, 1966, t. I, p. 327 sq.; L. KérY, Canonical Collections..., p. 61-71.

20. Lex romana Visigothorum, éd. G. HAENEL, Leipzig, 1849.

21. Alcuin se réfère notamment à : Mt 5, 7 ; Mt 9, 13 ; Lc 6, 36-37; Lc 7, 39 ; Lc 23, 34 ; 1 Co 4,$5 ;$ Ga 6,1 ; Jc 2,$13 ;$ Jc 3,$14 ; 1$ Jn 1,8 .

22. L. JÉGOU, L'Évêque, juge de paix. L'autorité épiscopale et le règlement des conflits (VIII -XI $^{e}$ siècle), Turnhout, 2011, p. 94.

23. 1 Jn 1, 8. Alcuin, Lettre 245, p. 394.

24. L'article de R. Meens ( Sanctuary, Penance, and Dispute Settlement under Charlemagne... », p. 297) souligne le rôle de la pénitence, facilitée par le temps mort garanti par le droit d'asile, dans le processus de réconciliation du réfugié avec la communauté.

25. Jc 2, 13. Alcuin, Lettre 245, p. 394. 
homélie de $399^{26}$, et le second, au début de la Cité de Dieu ${ }^{27}$, fondent de la même façon la protection des réfugiés - alors non positivement sanctionnée par la législation impériale - sur le devoir de miséricorde qui incombe à tout chrétien, et par conséquent aux détenteurs du pouvoir séculier. Chez Alcuin comme chez ces deux évêques, le droit d'asile ne repose donc pas sur une logique propre au droit humain, mais est justifié avant tout par la fidélité aux enseignements du Christ. De fait, toutes les citations d'Alcuin, à une exception près, sont issues ici du Nouveau Testament. Ce n'est que dans un second temps qu'il revient au droit séculier de formaliser une institution contraignant les individus à se conformer au devoir de miséricorde : c'est la raison d'être du droit d'asile, conçu comme une interface devant favoriser l'expression de la clémence et du pardon dans le règlement des conflits.

\section{L'asile comme outil de promotion de la miséricorde}

En invoquant le premier canon du premier concile d'Orléans, réuni en 511 à l'initiative de Clovis ${ }^{28}$, Alcuin souligne ainsi que le recours à l'asile des églises doit permettre aux criminels - meurtriers, adultères et voleurs - d'échapper à « la mort, la mutilation ou tout genre de peine » (de morte, de debilitate et omni poenarum genere), et que cette clémence doit leur être garantie par serment prêté par leur poursuivant.

Alcuin, cependant, ne cite pas le texte plus avant et occulte deux éléments importants : d'une part, la nécessité pour le réfugié de convenir avec son persécuteur d'une satisfactio, sans aucun doute d'ordre pécuniaire, et d'autre part l'obligation faite à ce dernier de respecter son serment, sous peine d'excommunication, et d'accepter la composition comme seule réparation du tort subi. Cette première altération opérée par Alcuin sur ses sources en appellera d'autres. Interprétées par Hélène Noizet comme une forme de «nettoyage », ces altérations seraient la preuve de la malhonnêteté de l'abbé, qui passerait ainsi sous silence les morceaux de lois qui contredisent sa vision du droit d'asile ${ }^{29}$.

En tronquant ce premier texte,Alcuin semble en effet présenter le droit d'asile comme une garantie d'impunité totale pour les réfugiés, protégés contre « tout genre de peine ». Selon $\mathrm{H}$. Noizet, cela revient à travestir le sens des dispositions sur l'asile contenues dans le Bréviaire d'Alaric et les conciles mérovingiens, car celles-ci « comprenaient manifestement

26. Jean Chrysostome, Patrologia Graeca,t. 52, p. 391-396. En dépit de cette ressemblance, il n'est pas certain qu'Alcuin ait compté Jean Chrysostome au nombre de ses sources.

27. Augustin d’Hippone, De Civitate Dei, I, viI (La Cité de Dieu. Livres I-V, éd. G. BARdy, G. Combès, B. Dombart et A. Kalb, Paris, 1959, p. 209).

28. Concilium Aurelianense a. 511, c. 1, éd. F. MaAssen, MGH, Concilia, I, Hanovre, 1893, p. 2 ; Les Canons des conciles..., t. I, p. 70-73.

29. H. NoIzet, « Alcuin contre Théodulphe... », p. 119-120. Pour 1'auteur, Alcuin « réinvente » le droit d'asile par ses altérations. 
deux volets », le deuxième précisant les « limites » du droit d'asile et les « devoirs » incombant aux réfugiés.

Cen'est pourtant pas aussi évident, puisque certaines des constitutions majeures produites au Bas-Empire romain pour formaliser le droit d'asile ne se souciaient en fait guère de fournir le moyen de rétablir équitablement la paix entre le fuyard et son poursuivan $t^{30}$, laissant aux acteurs de la pratique judiciaire le soin de trouver une issue satisfaisante à chaque conflit. Ces textes visaient avant tout à garantir la protection des réfugiés contre toute violence. L'insistance d'Alcuin sur cette protection, aux dépens des outils de règlement du conflit, ne constitue donc pas une nouveauté.

Nous devons par ailleurs souligner que la nuance originellement incluse dans le canon de 511 n'est pas totalement absente de l'argumentaire de l'abbé, car celui-ci ne montre aucune réticence à la mentionner plus loin. En effet, le premier des deux canons apocryphes extraits par Alcuin de la Collectio Hibernensis apparaît comme un résumé du canon 1 du premier concile d'Orléans, et l'abbé n'en retranche pas le passage enjoignant les réfugiés à convenir d'une satisfaction avec leur poursuivant, preuve qu'il ne cherche pas à le masquer à tout prix. Dans le discours d'Alcuin, le rétablissement de la concorde entre les parties ne passe donc pas par l'amnistie du réfugié, car ce dernier peut être amené à négocier le rachat de sa faute auprès de son poursuivant. L'institution de l'asile doit cependant encourager l'expression de la miséricorde en écartant notamment le recours aux châtiments corporels.

Soucieux d'insister sur ce rôle de promotion de la miséricorde, l'abbé de Saint-Martin invoque par la suite ce qu'il désigne comme le vingtet-unième chapitre d'un texte placé sous l'autorité du pape Silvestre I ${ }^{\text {er }}$, mais qui correspond en fait au vingt-deuxième canon du cinquième concile d'Orléans de $549^{31}$. Ce texte exige des maîtres qu'ils prêtent serment de

30. Constitution du 21 novembre 419 (Sirm. 13) et constitution du 23 mars 431 (C. Th. IX, 45, 4) (Code théodosien I-XV. Code justinien. Constitutions sirmondiennes, éd. J. Rougé et R. Delmaire, Paris, 2009, p. 520-523 et 216-223).

31. Concilium Aurelianense a. 549, c. 22, MGH, Conc. I, p. 107 ; Les Canons des conciles..., t. I, p. 316-319. L. Wallach se base sur l'inversion entre les canons 21 et 22 de ce concile pour identifier la Collection canonique du manuscrit de Lorsch comme la source d'Alcuin, celle-ci présentant en effet la même confusion. Nous pouvons ajouter que cette collection est a priori la seule à présenter, dans le titre précédent les canons de ce cinquième concile d'Orléans, une constitution apostolique attribuée à Silvestre (en lieu et place de Zosime : voir F. MAASSEN, Geschichte der Quellen..., p. 249). Cela expliquerait la raison pour laquelle Alcuin place à tort notre canon sous l'autorité de ce pontife. Cependant, la Collection du manuscrit de Lorsch occulte le premier canon du concile d'Orléans de 511, pourtant correctement retranscrit par Alcuin. L. Wallach (Alcuin and Charlemagne..., p. 129) ne manque pas de remarquer cette difficulté mais la résout en signalant que ce texte précis est contenu dans la Collectio Hibernensis, laquelle constitue, comme nous l'avons indiqué, une autre source d'Alcuin. Néanmoins, cette dernière collection décontextualise ledit canon et on comprend mal comment l'abbé de Saint-Martin aurait pu savoir qu'il s'agissait du "premier chapitre des canons d'Orléans » (in canone Aurelianensi, capitulo primo legitur) s'il n'avait disposé de sources autres que les deux citées précédemment. 
pardonner leurs esclaves réfugiés dans une église et de ne les soumettre à aucun «supplice», quelle que soit leur faute, sous peine d'excommunication.

Une nouvelle fois, Alcuin élude la suite du canon, qui autorise notamment le maître à saisir légalement son esclave une fois le pardon garanti. Comme il l'avait fait avec le premier concile d'Orléans, l'abbé conserve seulement le passage enjoignant à ce que la miséricorde conduise, via l'institution de l'asile, à la mise de côté de tout châtiment corporel. H. Noizet souligne ici encore l'altération du texte ${ }^{32}$ : selon elle, cette disposition autoriserait en réalité "les maîtres » à saisir leurs hommes réfugiés dans une église et à les soumettre, une fois jugés et reconnus coupables, aux « mêmes châtiments que les autres ». Ce serait une nouvelle preuve des manipulations de l'abbé et donc de ses torts dans cette affaire.

Il nous faut cependant insister sur le fait que ce canon de 549 interdit explicitement tout châtiment. Au reste, ces dispositions n'ont de toute façon aucune raison de s'appliquer dans le cas du clerc d'Orléans, car elles ne concernent pas tous les hommes mais seulement les servi, catégorie à laquelle n'appartient manifestement pas le réfugié, quel que soit le sens que les hommes du IX ${ }^{e}$ siècle aient pu donner au terme servus.

C'est pourtant Alcuin lui-même qui entretient la confusion sur le statut du clerc fugitif. Dans sa volonté de mettre l'accent sur le nécessaire pardon des réfugiés et le rejet des châtiments corporels, l'abbé recourt à un texte hors de propos, afin de faire bénéficier le clerc d'Orléans de dispositions anciennes protégeant les esclaves de la fureur de leurs maîtres ${ }^{33}$. Dans la même optique, Alcuin invoque, dans sa lettre destinée à l'évêque inconnu ${ }^{34}$, un passage du Deutéronome exhortant le juste à héberger tout esclave qui se réfugierait auprès de lui pour tenter d'échapper à son maître ${ }^{35}$. Le tort de l'abbé est donc moins de faire mentir la source qu'il utilise que de se raccrocher, dans ce cas, à un texte inadapté, bien qu'il soit porteur de la même invitation à faire preuve de miséricorde via le respect du droit d'asile.

\section{Une procédure excluant toute violence}

Le principal grief formulé par Alcuin envers Théodulfe et ses hommes est leur mépris de cette miséricorde ${ }^{36}$ et l'emploi de la force pour saisir le clerc réfugié. L'entière responsabilité des violences survenues en ce dimanche est rejetée sur le camp adverse et l'abbé entend démontrer qu'ils

32. H. NoIzet, « Alcuin contre Théodulphe... », p. 120.

33. Ces dispositions mérovingiennes reprenaient en substance la règle établie par la constitution romaine du 28 mars 432 (C. Th. IX, 45, 5), transmise par le Code théodosien mais pas par le Bréviaire d'Alaric (Code théodosien..., p. 222-225).

34. Alcuin, Lettre 246, p. 399.

35. Dt 23, 15-16.

36. Alcuin regrette que, dans cette affaire, le jugement, rendu par Théodulfe, ait prévalu sur la miséricorde, en contradiction avec ce que préconise la Bible (Jc 2, 13) : Lettre 245, p. 394. 
ont ainsi, non seulement provoqué le désordre, mais qu'ils ont également enfreint plusieurs règles de procédure.

En s'appuyant sur le quatrième concile d'Orléans de $541^{37}$, Alcuin rappelle que la violation du droit d'asile, par la force ou la tromperie, est sévèrement punie d'excommunication. Le violateur, qualifié d'ennemi de l'Église, est alors tenu de rendre le réfugié à l'église et de faire pénitence pour son infraction. Il semblerait que l'intérêt d'Alcuin pour ce canon réside également dans la mise en avant de la nécessité de consulter le prêtre (sacerdos) ou celui qui a la charge (praepositus) de l'église avant d'entreprendre la moindre action. L'abbé tenterait ainsi de faire admettre que la procédure prévue par la législation conciliaire n'a pas été respectée : bien que l'évêque Joseph ait apporté sa caution à la saisie du réfugié, c'est le consentement d'Alcuin, en tant qu'abbé de Saint-Martin, qui aurait dû être requis. Dans tous les cas, l'usage de la violence demeure inadmissible.

C'est la même position que soutient Alcuin en recourant au deuxième canon apocryphe issu du pseudo-concile d'Agde ${ }^{38}$. Selon ce texte, les fidèles qui se réfugient dans le sein d'une église ne peuvent en être expulsés, mais doivent au contraire recevoir un traitement convenable jusqu'à leur jugement. On constate, ici encore, que la dénonciation de l'usage de la force n'exclut pas la nécessité de trouver une issue au conflit, via la mise en jugement des réfugiés. Alcuin n'occulte pas ce passage, ce qui prouve à nouveau qu'il ne promeut pas l'idée d'une amnistie des demandeurs d'asile, contrairement à ce qui a pu être affirmé. Son objectif demeure la défense d'une institution qui doit protéger les réfugiés de la violence, tout en encourageant l'expression de la miséricorde à leur égard au moment de la nécessaire résolution du litige.

À l'issue de son exploration de la législation conciliaire, Alcuin entend appuyer sa vision des procédures devant structurer l'institution de l'asile sur trois textes de droit romain ${ }^{39}$.

Il s'agit tout d'abord pour l'abbé de dénoncer à nouveau l'extraction violente des individus réfugiés dans une église, en se fondant sur la constitution promulguée le 23 mars 431 dans la partie orientale de l'Empire ${ }^{40}$, dans laquelle il faut reconnaître la source principale du premier canon du concile d'Orléans de 511. Cette loi affirme l'interdiction absolue d'user de violence envers les demandeurs d'asile et propose, par pragmatisme et dans un souci de préservation des parties les plus sacrées de l'église,

37. Concilium Aurelianense a. 541, c. 21, MGH, Conc. I, p. 92 ; Les Canons des conciles..., t. I, p. 278-279. Ce canon est cité dans son intégralité.

38. Via la Collectio Hibernensis : cf. supra.

39. La connaissance de ces fragments lui vient manifestement du Bréviaire d'Alaric puisqu'il cite les différentes interpretationes de ce recueil.

40. C. Th. IX, 45, 4 (Code théodosien..., p. 216-223) ; Lex romana Visigothorum, IX, 34, 1,p. 208-210. 
d'étendre la zone de protection à tous les espaces et bâtiments situés au sein des murs d'enceinte du sanctuaire ${ }^{41}$. Dès lors, la simple irruption violente des hommes de Théodulfe dans l'enceinte de l'abbaye avec le projet d'en extraire le fugitif était déjà condamnable.

Dans la même optique, Alcuin n'avait pas omis de souligner que les intrus avaient choisi un dimanche pour saisir le clerc réfugié. Il est certain que cette insistance devait mettre en évidence, en premier lieu, le contraste entre la violence mise en œuvre et l'attitude qu'il aurait été convenable d'observer en ce jour dédié à Dieu, lors duquel la miséricorde, le pardon des offenses et la célébration de la concorde devaient primer. L'abbé de Tours tente d'étayer cette position en se référant à une constitution du 25 janvier 409 qui, sous l'influence de la morale chrétienne, imposait aux juges d'exprimer, chaque dimanche, une clémence particulière à l'égard des prisonniers, en les autorisant à sortir de leur geôle afin qu'ils se rendent aux bains et collectent de la nourriture ${ }^{42}$. C'est avec quelque difficulté qu'Alcuin rattache à l'affaire cette loi, sans lien avec l'asile, en insinuant que le réfugié, ancien prisonnier de Théodulfe, n'aurait pas dû être violenté en ce dimanche.

L'abbé de Tours développe cette idée en affirmant que l'appel à l'empereur formulé par le clerc fugitif aurait dû le protéger de toute persécution jusqu'à ce qu'il puisse présenter son cas devant cette juridiction supérieure. Cet argument, autour duquel le débat va se cristalliser, est fondé sur une des sentences attribuées au juriste romain Paul, formulée sur la base de la Lex Iulia de vi publica ${ }^{43}$, qui a trait au droit d'appel (appellatio) à l'empereur des citoyens romains. Elle établit, entre autres, que tout individu convaincu de mauvais traitements sur un citoyen ayant fait appel à l'empereur encourt la peine capitale ou la déportation. C'est une manière pour Alcuin de signifier que cette forme de négation du recours à la justice de l'empereur est plus dommageable à l'autorité de Charlemagne que la résistance légitime des moines de Tours, la preuve étant que le droit romain punissait très sévèrement cette entorse aux procédures. De nouveau cependant, les spécificités de la législation de l'asile disparaissent derrière une loi sans rapport avec celui-ci ; l'empereur saura exploiter cette faille.

41. L'interpretatio du Bréviaire d'Alaric résume en évoquant les atria des sanctuaires, terme qui s'imposera au premier concile d'Orléans pour désigner l'ensemble de l'espace entourant l'église et servant de zone de refuge. Sur la signification d'atrium à l'époque mérovingienne, voir N. GAUTHIER, «Atria et portiques dans les églises de Gaule d'après les sources textuelles », dans C. SAPIN éd., Avant-nefs et espaces d'accueil dans l'église entre le IV et le XII siècle, Paris, 2002, p. 30-36.

42. Lex romana Visigothorum, IX, 2, 3, p. 174.

43. Lex romana Visigothorum, Pauli Sententiarum, V, 28, 1 sententia et 2 interpretatio, p. 438. 


\section{L'édification de l'empereur}

Si l'énumération de sources normatives constitue le cœur de son argumentaire, Alcuin encadre également sa synthèse de droit romain de deux récits dont l'exemplarité doit inciter l'empereur à prendre les bonnes décisions. Il adopte ce faisant l'attitude du pasteur qui illustre son enseignement par des histoires choisies pour leur caractère édifiant. Le contenu de ces deux sources quitte alors la sphère juridique pour pénétrer dans le genre apologétique. Si Marie-Céline Isaïa a pu démontrer que les récits hagiographiques pouvaient être sources de normes au Moyen $\hat{A}_{\text {ge }}{ }^{44}$, il semble que ces derniers n'occupent qu'une place secondaire dans l'argumentaire d'Alcuin, car ils visent essentiellement à présenter deux souverains, l'empereur Constantin et le roi Alaric, passant pour avoir témoigné un respect absolu du droit d'asile, institution fondée sur la loi divine et sur un droit romain se conformant à celle-ci.

Dans un premier temps, l'abbé cherche à mettre en évidence le caractère extraordinaire du droit d'asile, en certifiant que l'empereur Constantin lui-même aurait le premier, sous l'influence de Silvestre Ir conféré aux églises consacrées la capacité de défendre n'importe quel réfugié menacé par le danger d'un jugement (a iudicis periculo, qui in praesenti fuerit, defensetur). Cette disposition, particulièrement favorable aux demandeurs d'asile, est cependant apocryphe. Alcuin l'extrait des Actus Silvestri ${ }^{45}$, texte hagiographique progressivement élaboré entre les $\mathrm{V}^{\mathrm{e}}$ et $\mathrm{VI}^{\mathrm{e}}$ siècles et jouissant d'une très grande diffusion ${ }^{46}$. Silvestre y est présenté comme l'acteur principal de la conversion de Constantin, lequel aurait promulgué, dans les jours suivant son baptême, une série de onze lois favorables à l'Église, jusqu'alors persécutée par l'empereur ${ }^{47}$. Les Actus Silvestri sont ainsi construits sur une opposition entre le tyran païen, tourmenteur de la foi chrétienne, et le bon souverain, que le baptême a

44. M.-C. IsAïA, "Normes et hagiographie dans l'Occident latin ( $\mathrm{V}^{\mathrm{e}}-\mathrm{XVI}^{\mathrm{e}}$ siècle) », BUCEMA. Bulletin du Centre d'études médiévales d'Auxerre, 15 (2011), p. 229-236.

45. Sanctuarium seu Vitae sanctorum, éd. B. Mombritius, vol. II, Paris, 1910, p. 513.

46. T. Canella, Gli Actus Silvestri : genesi di una leggenda su Costantino imperatore, Spolète, 2006.

47. Notons, à la suite de Pierre Timbal (Le Droit d'asile..., p. 181), que la volonté de faire de Constantin le créateur du droit d'asile se retrouve dans la Lex Romana Curiensis, qui attribue à cet empereur la constitution de 431 (Lex Romana Raetica Curiensis, IX, 34, éd. K. Zeumer, MGH, Leges V, Hanovre, 1875-1889, p. 378-379). La Lex Romana Curiensis est un abrégé à diffusion restreinte (cinq manuscrits conservés) du Bréviaire d'Alaric, élaboré dans la région de Coire au début du $\mathrm{VIII}^{\mathrm{e}}$ siècle (Bibliotheca legum, en ligne : éd. K. UBL, D. Trump et D. Schulz, Université de Cologne, 2012-2016, http://www.leges.uni-koeln.de/ en/lex/lex-romana-curiensis/). Alcuin fournit pour sa part la bonne attribution, ce qui confirme qu'il a directement eu recours au Bréviaire d'Alaric. 
transformé en défenseur et promoteur des prérogatives de l'Église ${ }^{48}$. Ce passage veut montrer que la législation d'un grand monarque peut, en accord avec l'enseignement divin, soustraire n'importe quel réfugié au jugement des hommes, ou du moins suspendre le cours ordinaire de la justice terrestre. En se référant ainsi au premier empereur chrétien, l'abbé de Tours entend surtout fournir à Charlemagne, récemment couronné empereur, le plus prestigieux des modèles à suivre.

Alcuin certifie par la suite que même le « roi païen » Alaric a su respecter le refuge des églises chrétiennes au moment de son assaut sur Rome en 410. L'abbé se fonde sur l'œuvre de Paul Orose ${ }^{49}$, publiée en 416, qui rapporte comment les Wisigoths, gagnés au christianisme arien, épargnèrent les églises sur l'ordre de leur roi Alaric. Il convient d'expliquer que le récit d'Orose reprend celui de son ami saint Augustin ${ }^{50}$, et qu'il l'insère à la fin de son histoire apologétique du peuple chrétien pour démontrer comment la foi des Wisigoths dans le Christ fut seule capable d'adoucir leur sauvagerie barbare. C'est cette révérence envers Dieu qui, selon Orose, incita l'ennemi à préserver tout à la fois les individus réfugiés dans les églises de la ville et la vaisselle d'or destinée à l'office divin.

Alcuin reprend la démonstration à son compte : il qualifie le chrétien Alaric de «paganus rex », pour accentuer la barbarie supposée du roi des Wisigoths, et livre cet exemple à Charlemagne pour l'inciter à ne pas montrer moins de respect envers le refuge des églises que ce souverain «païen ». Le fait que la révérence des Wisigoths se soit particulier exprimée à l'égard des basiliques des apôtres Pierre et Paul est, de plus, censé toucher directement Charlemagne, du fait de sa dévotion particulière envers Saint-Pierre de Rome ${ }^{51}$.

\section{Les sanctuaires comme lieux de refuge traditionnels}

Alcuin cherche alors à conclure son plaidoyer en tentant de démontrer que, de manière coutumière, les sanctuaires ont toujours été des lieux de refuge protégés par le divin.

Invoquant la Bible dans l'espoir de neutraliser tout questionnement sur l'autorité des sources normatives citées précédemment, Alcuin soutient qu'il est possible d'y trouver des informations sur les « cités de fugitifs »

48. F. Monfrin, « La conversion du roi et des siens », dans M. Rouche éd., Clovis : histoire \& mémoire. Actes du Congrès international d'histoire de Reims (19-25 septembre 1996), Paris, 1997, p. 293-313.

49. Paul Orose, Historiarum adversum paganos, VII, 39 (PAul Orose, Histoires contre les païens, éd. M.-P. ArnAud-Lindet, Paris, vol. III, 1991, p. 113-115).

50. Augustin d’Hippone, De Civitate Dei.

51. Eginhard, Vita Karoli, cap. XXVII (éd. O. Holder-EgGer, MGH, SS rer. Germ., XXV, Hanovre/Leipzig, 1911, p. 32) ; Divisio regnorum, cap. 15 (éd. A. Boretius, MGH, Capit. I, Hanovre, 1883, p. 129). Voir R. SchiefFer, « Charlemagne and Rome », dans J. M. H. Sмітн éd., Early Medieval Rome and the Christian West, Leyde, 2000, p. 279-295. 
(de fugitivorum civitatibus), autrefois établies, à la demande de Dieu luimême, pour la protection des criminels et des pécheurs. L'abbé fait ici référence aux cités-refuge évoquées dans l'Ancien Testament et dont le texte biblique attribue la fondation à Moïse et à son successeur Josué. Au nombre de six, ces cités devaient permettre à l'homicide involontaire, et seulement à celui-ci, d'être protégé de la vengeance privée ${ }^{52}$.

Curieusement, Alcuin relègue ces dispositions de l'Ancien Testament aux côtés de semblables droits de refuge anciens, à l'exemple de celui que le mythe de la fondation de Rome attribue à Romulus ou de ceux que les temples païens du monde gréco-romain pouvaient conférer. À chaque fois, cependant, l'abbé reste évasif. Peut-être demeure-t-il incertain du champ d'action réel de ces droits antiques et préfère-t-il ne pas s'y attarder pour ne point prêter flanc à la contradiction ${ }^{53}$. La question de la possible application de ces exemples au cas de Tours ne concerne pas vraiment Alcuin ; il s'agit alors surtout, pour lui, de mettre en évidence un déséquilibre : comment les églises du Christ, Dieu véritable, pourraient-elles constituer un refuge moins sûr que les autres sanctuaires en demeurant les seules à ne pas protéger les fugitifs ${ }^{54}$ ?

Cette réflexion ne peut manquer de faire penser au huitième canon du concile de Mâcon de $585^{55}$, selon lequel il était impensable de ne pas manifester envers le refuge des églises un respect au moins équivalent à celui que les empereurs romains avaient imposé à l'égard de l'asile conféré

52. Ex 21, 13-14 ; Nb 35, 6-32 ; Dt 4, 41-43 ; Dt 19, 2-13 ; Jos 20, 1-9. Une partie de ces références scripturaires est transmise par la Collectio Hibernensis, au livre XXVIII : $c f$. H. WASSERSCHLEBEn, Die Irische..., p. 94-98.

53. Notons, par exemple, que Ex 21, 14 ordonne que l'homicide volontaire soit arraché de l'autel de Dieu pour être exécuté. Il est risqué pour Alcuin d'invoquer l'Ancien Testament puisque le droit d'asile ne se fonde en fait pas sur les normes vétérotestamentaires. C'est le propre de la Bible, qui demeure une «bibliothèque » de récits «souvent contradictoires » dans leurs conceptions de la justice : $c f$. L. JÉGOU, L'Évêque..., p. 95.

54. Dans la construction de son opposition entre les églises chrétiennes et les sanctuaires païens, Alcuin mentionne " l'asile de Junon ». Nous sommes alors tentés de dresser un parallèle avec l'évocation, dans une tout autre optique, de ce même " asile » (Iunonis asylum) par Augustin, dans le quatrième chapitre de la Cité de Dieu (Augustin D'HipPone, De Civitate Dei, I, Iv, p. 202-203). Signalons que le lemme asylum n'est jamais employé par les législations romaines et franques pour qualifier le refuge des églises chrétiennes. Augustin lui-même réserve ce terme pour désigner la protection conférée par les temples païens et inspirée de l'asylie grecque (A. Ducloux, "Ad ecclesiam confugere ». Naissance du droit d'asile dans les églises (IV'-milieu du ve s.), Paris, 1994, p. 139). Le vocable asylum est par conséquent très rare dans les sources du haut Moyen Âge occidental qui lui préfèrent la périphrase ad ecclesiam confugere, traduction de la formule grecque utilisée dès le concile de

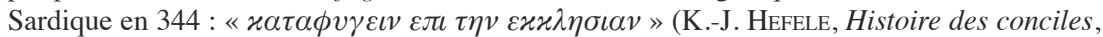
t. I.2, éd. et trad. H. LeCLercQ, Paris, 1908, p. 783). La présence du mot chez Alcuin tend à confirmer qu'Augustin est à compter au nombre de ses sources et qu'il lui sert de modèle dans sa célébration du refuge des églises chrétiennes, ce que nous avions suggéré plus haut.

55. Concilium Matisconense a. 585, c. 8, MGH, Conc. I, p. 168 ; Les Canons des conciles..., t. II, p. 466-467. 
par leurs statues ${ }^{56}$. De la même façon, le premier chapitre du capitulaire De partibus Saxoniae de 785 exige que les églises bâties en Saxe soient dûment respectées et qu'on ne leur reconnaisse pas moins d'honneur qu'aux temples païens ${ }^{57}$.

\section{La réponse de Charlemagne}

\section{Un argumentaire fondé sur le droit romain}

Celui qui fut chargé de répondre au nom de Charlemagne s'employa à transmettre le courroux de l'empereur. Sa collection de références implicites au Bréviaire d'Alaric démontre également sa maîtrise du droit romain.

Après avoir rappelé l'envoi aux moines de Tours d'un mandement leur ordonnant de livrer le clerc réfugié, le pli impérial souligne avec réprobation la colère qui émane du rapport d'Alcuin. La colère est en effet un péché dénoncé par l'apôtre Paul, comme le rappelle en 789 le chapitre 82 de l'Admonitio generalis $s^{58}$, et le droit romain rejette déjà toute accusation portée sous le coup de l'énervement ${ }^{59}$, une mesure réactualisée par le capitulaire De examinandis ecclesiasticis ${ }^{60}$. Si le ton de la lettre est lui-même particulièrement sévère, on constate néanmoins que son rédacteur prend soin de ne jamais diriger ses reproches vers la personne même d'Alcuin et prend plutôt le parti de n'accabler que les moines de Saint-Martin, ainsi accusés d'avoir semé la discorde " parmi les sages et les docteurs de l'Église » (inter sapientes et doctores ecclesiae) ${ }^{61}$.

Se fondant de nouveau sur le droit romain ${ }^{62}$, la missive ajoute que les doléances du réfugié envers l'évêque d'Orléans sont irrecevables car un criminel présumé n'est pas admis à accuser autrui, sa propre innocence devant au préalable être prouvée. Le clerc réfugié ayant confessé ses crimes ${ }^{63}$ et s'étant vu condamné pour ceux-ci, toute plainte de sa part apparaît illégitime. En 789, l'Admonitio generalis avait réaffirmé ces dispositions, précisant notamment qu'un criminel ne pouvait accuser une personne de statut supérieur ou un évêque ${ }^{64}$. Les efforts déployés par l'abbé pour faire

56. C. Th. IX, 44, 1 (Code théodosien..., p. 210-211). En 529, le Code justinien avait réaffirmé les modalités de ce droit de refuge particulier dans l'Empire byzantin : Corpus iuris civilis, I, 25, éd. H. Hulot, J.-F. Berthelot, P.-A. Tissot et A. Berenger, Metz, 1803, p. 199.

57. Capitulatio de partibus Saxoniae (785), cap. 1, MGH, Capit. I, p. 68.

58. Admonitio generalis (789), cap. 82, MGH, Capit. I, p. 61.

59. Lex romana Visigothorum, IX, 1, 3, p. 168-170.

60. Capitula de examinandis ecclesiasticis, cap. 7, MGH, Capit. I, p. 110.

61. Alcuin, Lettre 247, p. 400.

62. Lex romana Visigothorum, IX, 1, 7, p. 170.

63. Ce sur quoi Alcuin insiste : Lettre 246, p. 399.

64. Admonitio generalis (789), cap. 45, MGH, Capit. I, p. 56-57 ; Synodus Franconofurtensis (794), c. 36, MGH, Capit.I, p. 77. 
reconnaître son réfugié comme un pécheur, dont la confession aurait dû lui garantir miséricorde et pardon, se retournent contre lui.

Charlemagne s'appuie alors sur la rigueur du droit romain envers les criminels convaincus pour balayer l'argumentaire d'Alcuin. Il se saisit de l'analogie formulée par l'abbé entre la situation du réfugié de Tours et un passage des actes des apôtres dans lequel Paul, accusé par l'orateur Tertullus, est autorisé à faire appel à l'empereur pour exposer sa version des faits ${ }^{65}$. Charlemagne réfute la comparaison en retournant les armes d'Alcuin contre lui. Il se réfère à la deuxième partie de la loi romaine sur le droit d'appel ${ }^{66}$, précédemment citée par l'abbé mais également délestée par celui-ci des dispositions contredisant sa position. Dans la version complète de cette sentence en effet, le droit d'appel se voyait refusé de manière explicite aux criminels s'étant confessé ou ayant été condamnés puis incarcérés ${ }^{67}$. L'empereur souligne alors le fait qu'à la différence de Paul, «ce clerc infâme » (infamis clericus) était pour sa part déjà accusé, jugé et mis en prison.

Luitpold Wallach et Hélène Noizet ont vu dans cette nouvelle altération des sources la preuve de la malhonnêteté de l'abbé de SaintMartin $^{68}$. Si l'omission est effectivement trop importante pour paraitre innocente, constatons toutefois qu'Alcuin n'avait pas besoin de travestir les textes pour défendre correctement sa position. Les lois traitant directement de la question de l'asile suffisaient à son argumentaire. La sentence sur l'appel ne concerne quant à elle à aucun moment le droit d'asile, et précède d'ailleurs de plusieurs siècles la formulation de celui-ci ; cette sentence ne devrait dès lors pas même être discutée si l'on admet que l'asile est un privilège prévalant sur le reste de la législation. De fait, au Bas-Empire, le droit d'asile surpassait effectivement les lois ordinaires relatives à l'appel, car il avait pour principal objectif d'offrir aux réfugiés, même coupables ou déjà condamnés ${ }^{69}$, le bénéfice du soutien des évêques :

65. Ac 25, 10-12.

66. Lex romana Visigothorum, Pauli Sententiarum liber V, 28, 2, p. 438.

67. Le livre V des Sentences de Paul réaffirme ultérieurement l'irrecevabilité des appels formulés hors délai (alors fixé à cinq jours après le jugement) ou après des aveux. Lex romana Visigothorum, Pauli Sententiarum liber V, 35, 1, p. 440 et 37, 2, p. 442. Notons que, tout au long du Iv $v^{e}$ siècle, des constitutions romaines, transmises par le Code théodosien, ont réaffirmé, voire aggravé, les restrictions concernant l'appellatio: en 318, on interdit l'appel aux individus reconnus coupables des crimes dits graves que sont l'homicide, l'adultère, le maléfice et l'empoisonnement (C. Th. XI, 36, 1 = Brév. XI, 11, 1) ; l'interdiction sera étendue au crime de rapt, puis aux délits fiscaux, et régulièrement rappelée, notamment en 344 (C. Th. XI, 36, 7), 392 et 398 (C. Th. IX, 40, 15 et 16); dans les autres cas, l'appellatio peut être interjetée par le condamné dans les quelques jours suivant la sentence. Voir Code théodosien..., p. 200-205 et 276-281.

68. L. Wallach, Alcuin and Charlemagne..., p. 135 ; H. Noizet, "Alcuin contre Théodulphe...», p. 119.

69. Comme le préconise le concile de Sardique de $344:$ « [...] qui peccantes in exilio vel insulis damnantur, aut certe quamcumque sententiam excipiunt $\gg$ (C.-J. Hefele, Histoire des conciles..., p. 783). 
ces derniers pouvaient alors intercéder auprès de la cour impériale pour obtenir une remise de peine au nom de leurs protégés. La constitution du 27 juillet $398^{70}$, loi de circonstance sans postérité, promulguée à l'initiative d'Eutrope, favori de l'empereur Arcadius, pour restreindre la portée du droit d'asile et interdire aux clercs de soustraire des condamnés à l'application de leur sentence, avait elle-même admis la possibilité de déposer une provocatio, par souci d'humanité et dans la limite de trente jours après la condamnation $^{71}$. La loi de 419 , relative au droit d'asile, s'était inscrite dans la même veine en enjoignant à ce que « l'humanité infléchisse la justice ${ }^{72}$ », en réclamant que les réfugiés ne soient pas traités avec moins de pitié que les prisonniers, et en rappelant la capacité de l'évêque à intervenir, au nom de ces derniers, auprès du juge compétent, en fonction du droit ${ }^{73}$. Des voies de recours extraordinaires pouvaient donc subsister et l'institution de l'asile s'était superposée à celles-ci. La législation conciliaire mérovingienne avait d'ailleurs confirmé que la culpabilité ou les aveux d'un réfugié ne le privaient pas du droit d'asile ${ }^{74}$.

Dans cette optique, les efforts déployés par l'abbé de Tours afin de ménager à son réfugié un accès à la cour n'ont rien d'illégitime et, surtout, rien d'inédit. En définitive, cependant, cette insistance d'Alcuin sur le droit d'appel contribue surtout à déplacer l'objet de la discussion, élude la question du droit d'asile et permet à Charlemagne de ne répondre qu'à cet argument, d'autant plus fragile qu'il est le fruit d'une manipulation des sources. En mélangeant des textes de lois qui règlent des situations différentes, Alcuin entretient une confusion qui dessert son plaidoyer.

L'argumentaire produit par la cour impériale manifeste ainsi une bonne connaissance du droit romain, comme l'a démontré L. Wallach. À aucun moment, cependant, la législation particulière du droit d'asile n'est évoquée par la missive de Charlemagne. Il s'agit alors de déterminer si les lois sur l'asile devaient, ou non, prévaloir sur la législation ordinaire. On pourrait également se demander si, dans les faits, cette invocation du droit romain avait vraiment pour fonction d'imposer un règlement intransigeant du conflit, ou si elle en avait même la capacité.

70. C. Th. IX, 40, 16 (Code théodosien..., p. 202-205).

71. La provocatio se distingue ainsi de l'appellatio : c'est une supplique adressée aux autorités par des tiers (Code théodosien..., n. 4 et 6, p. 202-203 ; J. GAUDEMET, Les Institutions de l'Antiquité, Paris, 2002 [1967], p. 484).

72. Une formule qui ne manque de faire penser à Jc 2, 13, cité par Alcuin : Lettre 245, p. 394.

73. Sirm. 13 (Code théodosien..., p. 520-523).

74. Le canon 8 du deuxième concile de Mâcon (585) enjoignait les réfugiés à avouer leurs fautes à l'évêque pour qu'il puisse proposer une résolution du conflit respectueuse de l'église. Concilium Matisconense, c. 8, MGH, Conc.I, p. 168 ; Les Canons des conciles..., t. II, p. 466-467. 


\section{Charlemagne et le droit d'asile}

Le droit d'asile, tel qu'il est invoqué par Alcuin, vise à protéger les criminels des rigueurs de la justice afin qu'ils obtiennent miséricorde et pardon, ce qui peut interférer avec les procédures judiciaires ordinaires carolingiennes. Il s'agit d'un privilège qui implique la mise en place de mesures d'exception. Il est explicitement demandé à la victime d'une offense de faire preuve d'indulgence et de renoncer à tout ou partie du châtiment légitime. L'intransigeance ne peut plus avoir cours et les parties pénètrent sur le terrain de la négociation, de l'arbitrage, de la médiation ${ }^{75}$, car les solutions types ne sont fournies qu'à titre exceptionnel par les lois sur l'asile ${ }^{76}$. Comme l'indique le premier canon du concile d'Orléans de 511 , les parties, implicitement conseillées par les clercs, doivent convenir d'une satisfaction, en excluant « tout genre de peine ${ }^{77} »$.

La législation carolingienne, soucieuse, en théorie, d'universalité et d'intransigeance à l'égard du crime ${ }^{78}$, doit très tôt composer avec ce droit aux caractéristiques très particulières, motivé par la foi, fondé sur l'idée que la paix sociale s'obtient plus efficacement par la pratique de la charité et du pardon que par la mise en œuvre de la répression, celle-ci apparaissant d'ailleurs moins du ressort des hommes que de celui de Dieu, comme Alcuin se plaît à le rappeler ${ }^{79}$. Il s'agit dès lors de questionner le rapport de Charlemagne à la législation de l'asile, pour tenter de comprendre la logique qui sous-tend sa réponse à l'abbé de Tours.

75. Remarquons néanmoins que, dans la pratique, ces modes de règlements extrajudiciaires des conflits n'ont rien d'exceptionnels au haut Moyen Âge, même à l'époque carolingienne, et que les évêques sont régulièrement sollicités comme médiateurs ou arbitres pour rétablir la paix sociale. Voir, entre autres, R. LE JAN, «Justice royale et pratiques sociales dans le royaume franc au $\mathrm{IX}^{\mathrm{e}}$ siècle », dans La giustizia nell'Alto Medioevo, secoli IXXI, Spolète, 1997, p. 49 ; W. BRown, «Conflict, Letters and Personal Relationships in the Carolingian Formula Collections », Law and History Review, 25/2 (2007), p. 327, 337 et 341 342 ; L. JÉGOU, L’Évêque..., en particulier p. 16-18, 150-155 et 166-170. Ainsi, « la justice altimédiévale ne cherchait pas nécessairement à déterminer la légitimité de la cause et "rendre à chacun son dû", elle ambitionnait la réconciliation des parties, dans le respect des préceptes néotestamentaires » (L. JÉGOU, L'Évêque..., p. 86).

76. Seuls les codes de lois burgondes et wisigoths précisent la marche à suivre dans certains cas particuliers. Il s'agit du Liber constitutionum des Burgondes, titre LXX.2 relatif aux voleurs (éd. L. R. DE SALIS, MGH, LL nat. Germ., II.1, Hanovre, 1892, p. 96) ; de la Lex Romana Burgundionum, titre II.5-6 relatif aux homicides, (MGH, LL nat. Germ., II.1, p. 126-127) ; et du Liber iudiciorum des Wisigoths (éd. K. Zeumer, MGH, LL nat. Germ., I, Hanovre-Leipzig, 1902), dans des titres relatifs aux femmes adultères (III.2.2, p. 133-134), aux ravisseurs (III.3.2, p. 140-141), aux homicides (VI.5.16 (15), p. 281-282), aux parricides (VI.5.18 (17), p. 283) et aux déserteurs (IX.2.3, p. 367).

77. C'est-à-dire de toute peine afflictive. La suite du texte ouvre la voie au compromis, à la composition.

78. F.-L. Ganshof, « Charlemagne et l'administration de la justice... », p. 394-419.

79. Alcuin, Lettre 245, p. 394. 
En $785^{80}$, le capitulaire De partibus Saxoniae ${ }^{81}$ reprend dans son deuxième chapitre les dispositions d'Orléans I, mais impose la comparution du réfugié devant le plaid, tout en lui garantissant la commutation de tout châtiment corporel (mort et mutilation) en une amende à montant variable. Il indique également que le réfugié doit ensuite être conduit devant le roi pour que celui-ci en dispose à sa guise, bien qu'en toute clémence. Dans un esprit analogue, le chapitre 14 du même capitulaire ajoute, à la suite d'une liste de douze crimes punis de mort, que les individus ayant commis ces crimes capitaux en secret ont la possibilité de se confesser auprès d'un prêtre afin de se voir épargnés.

Si l'on ne devait retenir que ce texte, la législation carolingienne sur l'asile apparaîtrait cohérente : tout en réaffirmant les caractéristiques principales du droit d'asile romain - établi comme un temps mort permettant que s'exprime l'indulgence du poursuivant et des autorités -, Charlemagne intègre les aménagements conciliaires mérovingiens, mais insiste sur la nécessité d'insérer ce privilège dans une procédure publique, en systématisant la comparution des réfugiés devant un plaid qui décidera, comme la loi franque le préconise depuis plus de deux siècles et demi, de commuer toute peine afflictive en amende, par égard pour l'honneur de Dieu et des saints auxquels le réfugié a fait appel ${ }^{82}$.

Cette législation perd cependant en clarté lorsque l'on considère les dispositions contenues dans le capitulaire de Herstal de $779^{83}$. Celuici exclut en effet du droit d'asile les individus coupables d'une faute punie de mort par les lois (qui legibus mori debent). Les clercs ne doivent donc pas autoriser de tels criminels à pénétrer dans une église et, si ces derniers y parviennent malgré tout, il est proscrit de les fournir en vivres. Une telle disposition ne figure ni dans le droit romain ni dans les conciles mérovingiens.

Ces contradictions ont amené Pierre Timbal à formuler une hypothèse pour tenter de réconcilier ces lois ${ }^{84}$. Ainsi a-t-il proposé de distinguer, d'une part, les réfugiés uniquement accusés d'un crime mais non encore jugés, pour lesquels s'appliqueraient les dispositions du De partibus Saxoniae visant à les déférer devant le plaid, et, d'autre part, les réfugiés déjà condamnés

80. Selon la datation proposée par L. HALPHEN, Études critiques sur l'histoire de Charlemagne, Paris, 1921, p. 173-179, et confirmée par F.-L. GANsHOF, Recherches sur les capitulaires, Paris, 1958, p. 94.

81. Capitulatio de partibus Saxoniae (785), cap. 2, MGH, Capit. I, p. 68.

82. Ce contrôle systématique, par le plaid, des procédures de règlement des conflits est l'une des préoccupations de Charlemagne : R. LE JAN, « Justice royale et pratiques sociales... », p. 66-68.

83. Capitulare Haristallense (779), cap. 8, MGH, Capit. I, p. 48.

84. P. Timbal, Le Droit d'asile..., p. 141-143. 
par la justice, à l'endroit desquels aurait été rédigé le huitième chapitre du capitulaire de Herstal qui leur refuse le droit à l'asile. P. Timbal fonde son hypothèse sur le terme "reus », qui apparait dans ce dernier capitulaire et qu'il traduit, en rejetant toute équivoque, par « condamnés ».

Cette lecture des sources peut apparaître séduisante en ce qu'elle résout de manière commode la difficulté présentée par des lois a priori discordantes. Charlemagne aurait ainsi voulu interdire que le refuge des églises pût servir à annuler les condamnations déjà prononcées ${ }^{85}$.

Bien qu'il ne paraisse pas infondé de chercher à voir dans cette législation l'expression d'un certain idéal d'intransigeance de Charlemagne, il nous semble pourtant délicat de reconstruire, sur cette base fragile, une hypothétique théorie juridique distinguant le sort des accusés et celui des condamnés.

Il apparaît en premier lieu nécessaire de noter que le terme «reus » ne signifie pas systématiquement «condamné » mais peut également désigner une partie en cause dans un procès, un accusé dont la culpabilité n'est pas établie. Il reste ainsi difficile de savoir si le capitulaire de Herstal désigne uniquement les individus effectivement reconnus coupables d'un crime capital par un tribunal ou si la mesure s'étend aussi aux personnes seulement suspectées. De plus, le texte ne refuse pas le droit d'asile à tous les types de condamnés mais uniquement à ceux qui encourent la peine de mort. Ce manque de clarté fragilise ainsi toute tentative d'utiliser ce capitulaire pour étayer l'hypothèse de P. Timbal.

En ce qui concerne le capitulaire De partibus Saxoniae, il est vrai que ses deux canons relatifs au droit d'asile insistent sur la nécessité de conduire les réfugiés en procès, ce qui pourrait induire qu'on protège ici exclusivement des personnes non encore jugées donc non condamnées. Ce serait cependant postuler que les systèmes judiciaires anciens n'admettent aucun recours aux sanctions déjà prononcées et que les individus condamnés une première fois par un tribunal n'ont alors plus aucune option. Pourtant, nous avons vu que le droit d'asile s'était précisément construit comme une voie de recours extraordinaire, favorisant l'intercession des clercs auprès de l'autorité séculière dans le but d'atténuer une sentence ou de l'annuler complètement par l'action de la grâce ${ }^{86}$. Warren Brown souligne d'ailleurs

85. Cela manifesterait une volonté de faire respecter l'autorité de la chose jugée, notion déjà connue du droit romain et qui apparaît aussi comme l'une des préoccupations de Charlemagne. Rappelons néanmoins que les justices romaine et carolingienne connaissent malgré tout des voies de recours telles que l'appel à l'empereur ou la provocatio. P. F. GIRARD, Manuel élémentaire de droit romain, Paris, [1929] 2003, p. 1105-1115 ; F.-L. GANshoF, « Charlemagne et l'administration de la justice... », p. 404 et 16; J. GAUDEMET, Les Institutions..., p. 484.

86. Pour mémoire, dans la Rome républicaine, l'intercession correspond à un droit de veto. Elle est d'abord l'apanage des consuls qui peuvent par ce biais s'opposer unilatéralement à l'action de leur collègue. Le pouvoir d'intercession est par la suite également conféré aux tribuns de la plèbe qui peuvent dès lors, sur demande de n'importe quel citoyen, bloquer tout acte d'un magistrat au sein de Rome et dans ses alentours. $C f$. P. F. Girard, Manuel 
qu'à l'époque carolingienne nombre de conflits pouvaient donner lieu à une décision prise par un tribunal, mais ne trouver leur solution définitive qu'à l'occasion d'un compromis négocié ultérieurement, en dehors de toute procédure formelle. En analysant les Formules carolingiennes, W. Brown met en évidence le rôle, dans ce processus de pacification, de la supplique ou de l'intercession, adressée par voie épistolaire, notamment dans des cas de recours à l'asile ${ }^{87}$.

Rien, dans le De Partibus Saxoniae, ne s'oppose donc à ce que l'on définisse le droit d'asile chrétien comme cette voie de recours extraordinaire autorisant théoriquement quiconque, criminel convaincu ou simple innocent, à se placer sous la compétence directe de Dieu, de ses saints, ou de ses représentants terrestres - les évêques, le souverain - afin de réclamer la clémence et, le cas échéant, la révision de sa condamnation. Une voie de recours qui peut conduire à un nouveau procès, comme l'impose le capitulaire De partibus Saxoniae, ou à une procédure de médiation, voire d'arbitrage, telle que la propose le premier concile d'Orléans, afin de décider d'une remise de peine. En ce sens, le droit d'asile n'a aucune raison de constituer un blocage systématique des mécanismes de règlement des conflits. Ajoutons qu'il est question de sociétés chrétiennes pour lesquelles il n'y a rien d'inique à pardonner des pécheurs si l'on pense que cela peut les amener à la repentance et donc à la réhabilitation, l'essentiel demeurant le retour à la paix et à la concorde $^{88}$. Dans l'Occident médiéval, les élites chargées d'administrer la justice peuvent d'ailleurs se montrer soucieuses d'apparaître non seulement comme la source de la punition, mais également comme celle du pardon ; les deux rôles participent pleinement de la fonction judiciaire.

Est-il dès lors réellement nécessaire de recourir à une théorie affirmant que l'ensemble des condamnés sont exclus du droit d'asile par Charlemagne pour élucider les divergences entre le capitulaire de Herstal et le De partibus Saxoniae ? Compte tenu des sources à notre disposition, il paraît plus prudent d'admettre que rien ne l'expose clairement et de s'en tenir à une explication moins audacieuse.

On pourrait tout aussi bien considérer que le capitulaire de Herstal, en excluant de l'asile les individus passibles de la peine capitale, formule simplement une exception, ratione materiae, à la règle générale extraite de la législation conciliaire et rappelée par le capitulaire De partibus Saxoniae. Tout comme certains empereurs romains et certains souverains barbares avant lui, Charlemagne proclamerait ainsi, à l'aune d'une vision particulière de la justice, la trop grande gravité de certains crimes pour que

élémentaire..., p. 23 -24 et p. 1114 ; J.-P. Martin, A. Chauvot, M. Cebeillac-Gervasoni, Histoire romaine, Paris, [2001] 2010, p. 86.

87. W. Brown, «Conflict, Letters and Personal Relationships... », p. 327-328, 334 et 344.

88. L. JÉGOU, L'Évêque..., p. 84-93. 
leurs auteurs puissent bénéficier de la protection de l'asile ${ }^{89}$. C'est l'idée selon laquelle la préservation de la paix publique exige qu'on ne transige pas avec certaines fautes, au risque d'encourager le désordre. La question de l'équilibre entre le devoir chrétien de miséricorde, qui motive le droit d'asile, et les nécessités du maintien de l'ordre, qui légitiment, aux yeux du pouvoir, l'usage de la répression, n'est pas propre aux temps carolingiens ; elle sous-tend toute l'histoire du droit d'asile, chaque époque apportant ses propres réponses en fonction de paradigmes juridiques particuliers.

Notons toutefois que le capitulaire De partibus Saxoniae, bien qu'il se montre lui aussi très sévère contre toute atteinte à la paix, ne réitère jamais la même restriction de l'asile que le capitulaire de Herstal et ne présente que les dispositions ouvrant, de fait, ce droit à tous. Cet écart entre les deux textes amène donc plutôt à considérer l'influence du contexte de rédaction sur l'orientation des lois. La contradiction n'est en effet pas inhabituelle dans les législations du haut Moyen Âge et l'on peut aussi l'expliquer par le fait que les deux textes considérés ici sont les reflets de conjonctures différentes.

Les capitulaires sont le plus souvent des textes de circonstance $^{90}$. Celui de Herstal fut promulgué dans le contexte d'une période de troubles généralisés à l'ensemble du royaume. L'année 778 voit en effet l'échec de la campagne d'Espagne, le réveil de l'agitation en Aquitaine, l'intensification des incursions saxonnes, ainsi qu'un épisode de famine en Francie. Cela conduit le souverain à réaffirmer son autorité en édictant de strictes mesures pour garantir le maintien de l'ordre public. Tout le texte est empreint d'un durcissement et d'une sévérité motivés par la nécessité du redressement moral indispensable pour venir à bout des difficultés du temps. Cela pourrait expliquer l'intransigeance manifestée à ce moment précis à l'égard des auteurs de crimes capitaux, au point de nier leur droit à l'asile.

Le capitulaire De partibus Saxoniae fut quant à lui élaboré en 785, à la suite de la soumission et du baptême du chef saxon Widukind, après plusieurs années d'une guerre difficile. Le texte vise à imposer la conversion rapide de la Saxe et promulgue en ce sens de sévères sanctions contre toute atteinte portée à la religion chrétienne, aux édifices du culte ou aux clercs. Dans ce contexte, la réaffirmation du droit d'asile, privilège des églises du Christ, apparaît comme l'un des éléments du programme de christianisation de la région. Ajoutons qu'entre 779 et 785 l'optique des législateurs a pu évoluer, le capitulaire De partibus Saxoniae corrigeant la disposition du capitulaire de Herstal jugée a posteriori trop sévère et surtout en désaccord avec le droit canon. Cela expliquerait pourquoi le canon 2 du De partibus

89. Nous pensons notamment à l'exclusion des débiteurs du fisc par la constitution du 18 octobre 392 (C. Th. IX, 45, 1, Code théodosien..., p. 210-213), par l'Édit de Théodoric (Edictum Theoderici, cap. 71, éd. F. Bluhme, MGH, Leges V, p. 160) ou par le Liber iudiciorum (IX, 3, 4, MGH, LL nat. Germ., I, p. 380).

90. J. FAVIER, Charlemagne, Paris, 1999, p. 330 et 341. 
Saxoniae se révèle aussi proche du contenu d'Orléans I : il constituerait la simple reformulation carolingienne de dispositions canoniques anciennes ${ }^{91}$. Le droit canon y apparaît alors respecté, réaffirmé, mais se retrouve domestiqué par un pouvoir séculier qui se positionne comme le contrôleur de toute la procédure. L'essentiel n'est pas tant de discuter la légitimité de la clémence promue par le droit d'asile, que de déterminer quelle autorité sera chargée de contrôler sa mise en pratique. Le roi des Francs s'impose ainsi comme l'ultime recours et reprend, sciemment ou non, la place des empereurs romains dans l'administration du droit d'asile.

Les textes élaborés avant l'accession de Charlemagne à la dignité impériale sont ainsi très sensibles au contexte particulier de leur rédaction, ce qui déteint sur le contenu des mesures ayant trait au droit d'asile. Leur validité est limitée dans le temps comme dans l'espace, d'où la nécessité d'explorer la législation carolingienne ultérieure pour identifier les dispositions qui furent réellement confirmées par le pouvoir.

\section{Le droit d'asile dans l'affaire de Tours}

Il s'agit désormais de se questionner sur la logique juridique qui a prévalu dans l'affaire du clerc d'Orléans. Si le destin du réfugié nous reste en grande partie inconnu, la lettre de Charlemagne rend assez clairement compte du refus de la cour impériale de lui octroyer le bénéfice du droit d'asile. Pour autant, les raisons de cette position restent difficiles à appréhender.

Le contenu des différents textes invoqués par la missive impériale peut, de prime abord, laisser penser que le pouvoir n'a tout simplement pas voulu considérer cette affaire comme un cas d'asile, ce qui lui a permis de faire fi des lois protégeant les individus réfugiés dans une église. La fuite du clerc d'Orléans n'est pas perçue comme un appel légitime au droit, mais plutôt comme une nouvelle félonie appelant sanction. Le manque de précisions nous amène à privilégier cette idée d'une résolution autoritaire du litige, imposée par un souverain se hissant au-dessus des lois et ignorant les dispositions particulières du droit d'asile. Les sources suggèrent de toute façon que l'empereur focalisa finalement son attention sur la résistance des moines de Tours, la question de la soumission de chacun à son autorité lui important en définitive bien plus que le respect du droit d'asile. Cette détestation du désordre et du non-respect des hiérarchies, qui explique beaucoup sa position dans cette affaire, se révèle dans l'accusation de débauche qu'il formule à l'encontre des moines de Saint-Martinn ${ }^{92}$.

91. À l'exemple de ce que le roi des Francs entreprendra quelques années plus tard avec l'Admonitio generalis : J. FAVIER, Charlemagne, p. 338.

92. Alcuin, Lettre 247, p. 400. 
Si l'on veut malgré tout admettre que la législation de l'asile a été considérée par la cour avant la rédaction de la lettre aux moines de SaintMartin, deux hypothèses subsistent.

Le clerc aurait par exemple pu commettre une faute compromettant son droit à l'asile. Comme nous l'avons vu, la mise en place de restrictions quant au type de criminels admis à bénéficier de ce droit n'a rien d'inédit, et la législation carolingienne, via le capitulaire de Herstal, a ainsi pu souhaiter exclure de l'asile les auteurs de crimes capitaux ${ }^{93}$. Nous ignorons cependant la faute pour laquelle le fugitif fut en premier lieu emprisonné dans les geôles d'Orléans. Aucun des protagonistes de l'affaire ne prend la peine de rappeler les raisons de son incarcération première, comme si cela n'avait aucune incidence sur la logique à suivre pour résoudre la querelle. Il semble dès lors impossible de déterminer si le réfugié pouvait, ou non, se voir imposer pareille restriction. Ajoutons qu'il n'est pas certain que les dispositions du capitulaire de Herstal aient alors été considérées comme celles en vigueur au moment de l'affaire. La lettre de Charlemagne n'y fait pas allusion pour justifier son refus de voir le fugitif protégé par les moines de Tours, alors même qu'elle prend par ailleurs soin de se référer constamment aux législations romaine et carolingienne.

Une deuxième hypothèse se fonderait sur la théorie de Pierre Timbal ${ }^{94}$. Il est vrai que l'insistance de Charlemagne sur la condamnation effective du clerc d'Orléans pourrait conduire à considérer que c'est bien cette logique implicite qui a prévalu dans ce litige, le réfugié voyant son droit à l'asile nié par le simple fait qu'il se fût déjà trouvé sous le coup d'une condamnation. Nous avons cependant observé que le souverain cherche avant tout à contredire Alcuin en s'appuyant sur une loi romaine réglant le droit d'appel à l'empereur, un droit dont les condamnés sont explicitement exclus ${ }^{95}$. En invoquant uniquement cette disposition particulière, Charlemagne refuse de fait de considérer cette affaire comme une demande d'asile, c'est-à-dire comme une voie de recours extraordinaire disposant de sa propre législation et autorisant en théorie les individus jugés coupables à implorer une mitigation de leur peine. Pour l'empereur, la nature de l'affaire importe peu : il semble surtout saisir l'occasion de se raccrocher au premier texte juridique lui permettant de faire basculer

93. Il faudrait au préalable admettre que les dispositions de ce texte se sont imposées dans la pratique, au détriment des procédures anciennes rappelées par le De partibus Saxoniae, ce dont nous doutons. L'écart entre l'idéal répressif de certains capitulaires et la réalité de la pratique, volontiers tournée vers le compromis, a notamment été souligné par R. LE JAN, « Justice royale et pratiques sociales... », p. 59-61, 71-75 et 84-85.

94. Selon qui la législation de Charlemagne refusait le droit d'asile à tout individu déjà condamné, théorie dont nous avons souligné la fragilité : $c f$. supra.

95. S'ils ont avoué ou dépassé le délai prescrit. 
le rapport de force en sa faveur, stratégie qui n'a rien d'inhabituel dans la résolution des conflits au haut Moyen $\hat{\mathrm{A} g} \mathrm{e}^{96}$.

\section{Le capitulaire de 803}

Une vingtaine d'années après la promulgation du De partibus Saxoniae, de nouvelles dispositions relatives au droit d'asile apparaissent dans le capitulaire d'addition aux lois de 803.

Hélène Noizet comme Rob Meens ont voulu relier ce capitulaire à l'affaire de Tours, car le deuxième chapitre de ce texte règle la question particulière des criminels réfugiés au sein d'une immunité et précise les sanctions encourues par l'immuniste qui s'opposerait à leur saisie. Il s'agirait de la réaction législative de l'empereur à la résistance des moines de SaintMartin. L'affaire de Tours relève cependant du droit d'asile, non de l'immunité, privilège qu'aucune des deux parties n'invoque jamais. Barbara Rosenwein a d'ailleurs démontré que les législateurs carolingiens ne confondaient pas les deux domaines, en soulignant, à juste titre, que l'asile et la protection contre la saisie se voyaient au contraire réaffirmés dès le chapitre suivant de ce même capitulaire de $803^{97}$. Dès lors, s'il peut être possible de discerner dans ces dispositions le souci constant du pouvoir impérial de réaffirmer la nécessaire soumission de chacun aux représentants de son autorité, nous nous garderons d'y voir une tentative de restreindre le droit d'asile ${ }^{98}$.

Le troisième chapitre du capitulaire se conforme en réalité aux dispositions anciennes et reprend la constitution romaine de 431, déjà citée, en disposant que les personnes qui se réfugient sous le portique d'une église peuvent y demeurer à l'abri de toute violence sans qu'il ne leur soit même nécessaire de pénétrer dans l'édifice principal. Confirmant le De partibus Saxoniae, le législateur ajoute cependant que des hommes probes doivent amener le réfugié à avouer sa faute et sont invités à le conduire au plaid pour qu'il y soit jugé.

Le capitulaire n'apporte ainsi aucune réelle nouveauté en matière de droit d'asile et choisit de réaffirmer la mesure la plus classique, celle que le

96. Laurent Jégou montre, à travers plusieurs conflits des $\mathrm{X}^{\mathrm{e}}-\mathrm{XI}^{\mathrm{e}}$ siècles notamment, que l'on se situe dans un «contexte juridique d'internormativité », au sein duquel les parties peuvent invoquer, selon les circonstances, la norme qui se révèle la plus favorable à leur cause, et recourir à une multiplicité d'outils judiciaires ou extrajudiciaires pour parvenir à une issue satisfaisante (L. JÉGOU, L'Évêque..., p. 433-434).

97. B. Rosenwein, Negotiating Space : Power, Restraint and Privileges of Immunity in Early Medieval Europe, Ithaca, 1999, p. 227-228. Voir aussi les remarques proposées par G. BÜHRER-ThIERRY et L. JÉGOU, «Construction des pouvoirs et formation des espaces sacrés : le paradoxe de l'immunité. Autour de Negotiating Space », BUCEMA. Bulletin du Centre d'études médiévales d'Auxerre, hors-série 5 (2013), en ligne : http://cem.revues.org/12537 (consulté le 4 juillet 2016).

98. À la différence de H. NoIzeT, «Alcuin contre Théodulphe... », p. 124-125, et de R. Meens, « Sanctuary, Penance, and Dispute Settlement under Charlemagne... », p. 295. 
De partibus Saxoniae empruntait déjà au concile d'Orléans de 511. À nouveau, cependant, l'accent est mis sur l'importance de la comparution systématique du réfugié devant le tribunal public. Cela manifeste une fois encore la forte volonté du pouvoir carolingien de domestiquer les procédures du droit d'asile et de les soumettre étroitement au contrôle de la justice séculière.

Pour Hélène Noizet, cette prise en main rigoureuse de la législation de l'asile par un pouvoir impérial régénéré constitue une nouveautée ${ }^{99}$. Néanmoins, au Bas-Empire, l'empereur se positionnait déjà sans discussion au sommet de toute la procédure : c'est à lui que l'épiscopat adressait ses requêtes et c'est bien lui qui pouvait exceptionnellement décider de borner ce privilège, comme l'indique encore la constitution de 431 .

Subsiste toutefois une dernière difficulté. À une date proche de la promulgation du capitulaire de 803, Charlemagne fait mettre par écrit la Lex Saxonum ${ }^{100}$, dont un article renouvelle, pour les Saxons, la restriction établie par le capitulaire de Herstal : les condamnés à mort qui se réfugient dans une église ne doivent pas y trouver la paix ${ }^{101}$. On peine à comprendre ce court article, qui contredit le De partibus Saxoniae et le capitulaire de 803. Cette dissonance pourrait cependant être expliquée de nouveau par le contexte de production de la Lex Saxonum. La mise par écrit de celle-ci eut moins pour optique de recenser les lois devant s'appliquer en Saxe, que de rendre manifeste l'intégration de la région à l'Empire et l'on pourrait donc douter de la portée effective de ce texte dont la diffusion fut par ailleurs très faible ${ }^{102}$. Il est ainsi probable que sa rigueur à l'égard des auteurs de crimes capitaux soit avant tout le reflet d'une volonté d'assurer la pacification et la soumission totale de la Saxe, qui avait causé tant de difficultés à Charlemagne. En tout état de cause, les dispositions de ce texte ne pouvaient valoir que pour la Saxe, ce qui incite encore une fois à relativiser son impact à l'échelle de l'Empire.

Devant prouver à Charlemagne la légitimité du secours apporté par les moines de Tours à un clerc réfugié dans leur église, Alcuin extrait de sa bibliothèque deux corpus de lois qui ont fondé le droit d'asile chrétien : les constitutions impériales $\mathrm{du} \mathrm{v}^{\mathrm{e}}$ siècle, transmises par le Bréviaire d'Alaric, et les conciles francs du vi $\mathrm{e}^{\mathrm{e}}$ siècle. Ayant tout d'abord rappelé que le droit d'asile constitue une mise en application de l'idée chrétienne de miséricorde, l'abbé de Tours expose les textes romains et mérovingiens qui

99. H. NoIzEt, « Alcuin contre Théodulphe... », p. 126.

100. R. LE JAN, « Justice royale et pratiques sociales... », p. 63.

101. Lex Saxonum, cap. 28, éd. K. DE Richthofen, MGH, Leges V, p. 64.

102. Deux manuscrits conservés : Bibliotheca legum, éd. K. Ubl, D. Trump et D. Schulz, Université de Cologne, 2012-2016 [en ligne] : http://www.leges.uni-koeln.de/en/lex/lexsaxonum/ [consulté le 7 septembre 2016] ; Laurent Jégou doute du degré d'application réel de ce genre de lois (L. JÉGOU, L’Évêque..., p. 40-44). 
établissent ce droit comme une voie de recours extraordinaire autorisant les criminels à obtenir une mitigation de leur peine voire une grâce. Alcuin dévoile cependant les failles de son raisonnement lorsqu'il invoque des dispositions qui ne présentent aucune pertinence pour le litige en cours et nuisent au contraire à la solidité de son argumentaire.

Pour sa part, l'empereur ne considère jamais cette affaire comme un cas d'asile et ne se réfère donc pas à la législation particulière de ce droit. De manière quelque peu opportuniste, il s'appuie à son tour sur le Bréviaire d'Alaric pour rejeter le droit d'appel du réfugié et insiste particulièrement sur la nécessaire soumission de chacun à son autorité. Si les anciennes dispositions relatives à l'asile n'ont pas prévalu dans cette affaire, Charlemagne ne les a pas pour autant rejetées de sa législation. Malgré la promulgation, au tournant des $\mathrm{VIII}^{\mathrm{e}}$ et $\mathrm{IX}^{\mathrm{e}}$ siècles, de deux lois de circonstance refusant la protection de l'église aux auteurs de crimes capitaux, le souverain franc n'en réaffirme pas moins à plusieurs reprises la législation mérovingienne de l'asile, en la soumettant au contrôle de la justice séculière. Ces dispositions anciennes apparaissent non seulement dans les lois de 785 et de 803, mais également dans les canons du concile de Mayence de $813^{103}$ et dans un capitulaire ultérieur ${ }^{104}$; elles peuvent dès lors être regardées comme celles qui, en définitive, devaient s'imposer.

Maxence Bidu - Université Paris-Ouest Nanterre La Défense - UMR 7041, ED 395

\begin{abstract}
Alcuin, Charlemagne et le droit d'asile
Entre 801 et 802, une profonde querelle vient opposer Charlemagne et Alcuin au sujet d'un clerc d'Orléans réfugié dans l'abbaye Saint-Martin de Tours. L'empereur réclame que le fugitif soit livré à l'évêque Théodulfe, mais Alcuin s'y refuse, invoquant le droit d'asile. La dispute est l'occasion d'un intense échange épistolaire au cours duquel les deux parties exposent longuement leurs argumentaires, fondés chacun sur une recension détaillée des normes canoniques et séculières applicables. Le dossier juridique constitué par Alcuin revêt une importance particulière pour l'histoire du droit d'asile à l'époque carolingienne, car il permet d'identifier les sources disponibles au début du $I X^{e}$ siècle pour résoudre cette question, et illustre le processus de sélection des textes mis en œuvre par l'abbé. Cet article se propose d'analyser, dans le détail, les positions des deux principaux protagonistes et, ce faisant, de mettre en lumière le rapport ambivalent qui les lie. Malgré la relation privilégiée que partagent Charlemagne et Alcuin, il s'instaure à cette occasion un rapport de force et la question du droit d'asile, savamment défendu par l'abbé, disparaît alors derrière la démonstration d'autorité de l'empereur.
\end{abstract}

Alcuin - Charlemagne - droit canonique - droit d'asile - Saint-Martin de Tours

103. Réuni à la demande de l'empereur : Concilium Moguntinense, c. 39, éd.

A. Werminghoff, MGH, Conc. II.1, Hanovre-Leipzig, 1906, p. 271.

104. Capitula missorum, cap. 8, MGH, Capit. I, p. 182. 


\section{Alcuin, Charlemagne and Sanctuary}

Circa 801-802, a violent dispute regarding a cleric from Orléans seeking sanctuary in the basilica of St Martin in Tours opposed Charlemagne to Alcuin. The emperor demanded the cleric to be handed over to Bishop Theodulf. But Alcuin resisted this claim, pleading the respect of the right of sanctuary. The quarrel led to an important exchange of letters, an occasion for each part to present a developed argumentation, based on a thorough inventory of canonical and secular norms. Alcuin's plea appears to be a particularly significant material to study the history of Carolingian sanctuary, as it allows us to identify the sources related to this ecclesiastical privilege available at the beginning of the ninth Century. The approach adopted in this article is to provide a substantial analysis of each protagonist's stance on this matter, thus shedding some light on the nature of their relationship. Although they shared this special bond, this event caused a rather curt confrontation, which shaded the lying legal matter. The erudite defence of asylum produced by the abbot vanished when confronted to the emperor's display of authority.

Alcuin - asylum - basilica of St Martin - canon law - Charlemagne sanctuary - Tours 
